\title{
POST-AGB STARS AS EXTRAGALACTIC CANDLES
}

\author{
HOWARD E. BOND \\ Space Telescope Science Institute \\ 3700 San Martin Dr., Baltimore, MD 21218 USA
}

\section{Introduction}

The extragalactic distance scale depends heavily on one primary distance indicator: Cepheid variables. Given the conflict between stellar ages and the age of the Universe implied by $H_{0}$, it is worth checking the zero point with as many independent methods as possible.

I argue in this paper that the immediate progenitors of planetary nebulae $(\mathrm{PNe})$, i.e., stars in transition between the asymptotic giant branch (AGB) and planetary nuclei, may constitute a superb new class of standard stellar candles. These "post-AGB" (PAGB) stars are expected to have a narrow luminosity function in "Population II" systems, they should lie in environments that do not possess Cepheids or complicated interstellar extinction (ellipticals and halos of spirals), and they can be calibrated within the Milky Way. Thus PAGB stars should become an important new primary distance indicator, comparable in many ways to Cepheids.

My collaborators in this work are Laura K. Fullton, Abhijit Saha, and Karen Schaefer (all at STScI), and Rex Saffer (Villanova University).

\section{Stellar Candles in Old Populations}

Several well-known distance indicators are based on stars of low to intermediate mass, including RR Lyrae stars, long-period variables, non-variable AGB stars, and the luminosity of the red-giant tip. The planetary-nebula luminosity function (PNLF) technique is particularly well known to the present audience (see Jacoby 1996, and his contribution to this Symposium).

I follow Aaronson \& Mould (1986) in listing the criteria for a standard candle (to which I add parenthetical remarks that apply specifically to stellar candles): 


\section{POST-AGB STARS AS STANDARD CANDLES}

- Small scatter (e.g., a small range in $M_{V}$ )

- Available over a wide distance range (i.e., high luminosity)

- Minimal corrections (e.g., for reddening or metallicity)

- Objective measurables (e.g., stellar magnitudes)

- Physical basis (e.g., a basis in theoretical evolutionary tracks)

To these I add as additional desiderata:

- Easily recognizable objects (i.e., requiring a minimum of scarce telescope time)

- Calibratable within our Galaxy (i.e., primary distance indicators).

Although the various candles mentioned above have proven extremely useful, they do not in general satisfy all of these criteria. For example, RR Lyrae stars are not of high luminosity, and require a long time-series of telescope time. The absolute magnitudes of red giants, AGB stars, and PNe cover a wide range, so one must go deep enough, and detect enough objects, to recognize features in their luminosity functions (e.g., the turnover near the bright end of the PNLF). The PNLF in particular does not have a primary zero point established within the Milky Way, due to our poor knowledge of the distances of individual Galactic PNe (see the contributions of Terzian and Harris to this Symposium).

By contrast, I argue that PAGB stars arising from old populations, and passing through the proto-PN stage, satisfy all of the standard-candle criteria itemized above. They are thus a particularly "clean" candle, which will allow us to step out to the Virgo Cluster (using HST) on the basis of a remarkably small number of "rungs" in the distance ladder.

\section{PAGB A- and F-Type Stars as Standard Candles}

As a low- to intermediate-mass star ascends the AGB, the mass of its hydrogen-rich envelope decreases due to nuclear burning from below and mass loss from above. When the envelope reaches $\sim 10^{-2} M_{\odot}$, the star leaves the AGB and evolves rapidly across the HR diagram. In a few times $\sim 10^{4} \mathrm{yr}$ or less, $T_{\text {eff }}$ reaches $\sim 30,000 \mathrm{~K}$ and the surrounding AGB wind is ionized by stellar UV radiation, producing a $\mathrm{PN}$.

My proposed candles are the transition objects of spectral types A and $\mathrm{F}$, located between the AGB and the realm of PN nuclei. They lie in the approximate temperature range $5,000 \mathrm{~K}<T_{\text {eff }}<10,000 \mathrm{~K}$. These objects are often called proto-PNe (e.g., Hrivnak, this Symposium).

The top panel of Fig. 1 shows typical PAGB evolutionary tracks for several remnant masses (kindly provided by L. Stanghellini, based on the well-known Schönberner and Paczynski tracks). The stars evolve across the HR diagram at constant $L / L_{\odot}$, with higher luminosities corresponding to 


\section{H. Bond}
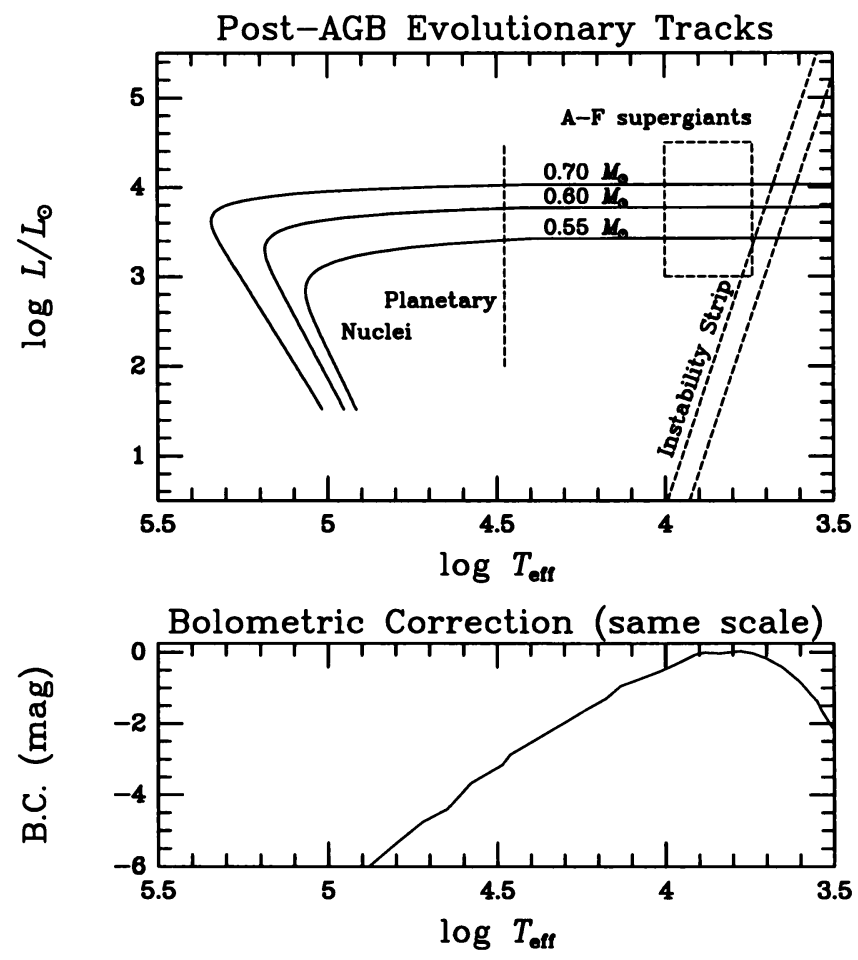

Figure 1. (Top). Schematic HR diagram showing PAGB tracks for three remnant masses, the pulsational instability strip, the location where ionization of the circumstellar wind creates a PN (vertical dashed line), and the location of the A- and F-type PAGB supergiants (dashed rectangle). (Bottom). The bolometric correction, plotted on same scale as the top panel, but in magnitude units. This curve shows the shape the PAGB tracks have in a plot of $V$ mag vs. $T_{\text {eff. Thus }}$ PAGB $A$ and $F$ stars are the visually brightest members of Population II.

higher remnant masses. Also shown schematically is the Cepheid instability strip. The dashed rectangle shows the approximate location of the A- and F-type PAGB stars, lying blueward of the instability strip.

The bottom panel of Fig. 1 plots the bolometric correction (from Kurucz models) against $T_{\text {eff }}$. Since the tracks are horizontal in $\log L / L_{\odot}$, this plot shows the shape of the tracks in the $V$ magnitude. Because the B.C. is smallest at late $\mathrm{A}$ and early $\mathrm{F}$ types, these are the visually brightest members of Population II.

Moreover, we expect the luminosity function (LF) for PAGB A and F stars to be quite narrow, for the following reasons. (1) A very sharp lower cutoff should exist, corresponding to the lowest-mass stars in the population which are currently leaving the main sequence. In an old population, such as the halo of a spiral, this cutoff will correspond to PAGB stars of $\sim 0.55 M_{\odot}$, which are the descendants of main-sequence stars of $\sim 0.8 M_{\odot}$. 
(2) The upper cutoff of the PAGB LF is set by the much shorter transition times for more massive remnants. These are a general property of the earlier calculations of PAGB evolution (Paczynski 1971; Schönberner 1983). Although the transition times are extremely dependent upon the adopted mass-loss laws and are thus rather uncertain, more recent calculations (e.g., Blöcker \& Schönberner 1990; Vassiliadis \& Wood 1994; Blöcker 1995) continue to show this property.

In a population containing only old stars, the PAGB LF should shrink almost to a delta function. In the context of measuring extragalactic distances, this means that we only have to detect the PAGB stars; it is not necessary to go much deeper than the detection, as is required in methods such as the PNLF.

\section{PAGB A and F Type Stars in the Field and Globular Clusters}

Many field PAGB A and F stars are now known in the solar neighborhood, e.g., from objective-prism surveys for high-latitude supergiants, or from optical identifications of IRAS sources. Well-known examples include $89 \mathrm{Her}, \mathrm{BD}+39^{\circ} 4926$, and HD 46703. Their $T_{\text {eff }}$ and $\log g$ values from high-resolution spectroscopic analyses are fully consistent with their lying on standard PAGB tracks (see Bond 1996). However, the spectroscopic $\log g$ values of the field PAGB stars are not accurate enough to calibrate their absolute luminosities, even if their masses were known a priori. Therefore it is necessary to base an empirical calibration on PAGB stars in globular clusters.

A few PAGB A- and F-type stars are in fact known in globular clusters, and can serve for a preliminary calibration of their absolute magnitudes. The best-known example is the 10th-magnitude A supergiant ROA 24 in $\omega$ Cen. Table 1 lists this object, along with three other candidates.

The table dramatically confirms our expectation that the PAGB stars in old populations will have a very narrow LF: the scatter in $M_{V}$ among the four stars is only $0.2 \mathrm{mag}$ - considerably less than the range among Cepheids at a given pulsation period. Moreover, the mean $M_{V}$ of -3.4 is in remarkable agreement with the luminosity of the lowest-mass $\left(0.546 M_{\odot}\right)$ PAGB track of Schönberner (1983).

NGC 5986 is particularly instructive. It contains two PAGB A stars (Bond 1977), and the fact that their $V$ magnitudes agree to within $0.09 \mathrm{mag}$ decisively argues for a very small range in absolute magnitudes. The distance for this cluster is based at present upon the old photographic photometry of Harris et al. (1976). However, L. Fullton, STScI summer students S. O'Brien and C. Marois, and myself are reducing CCD frames obtained with the CTIO 0.9 - and $1.5-\mathrm{m}$ telescopes. A preliminary color-magnitude 
TABLE 1. Absolute Magnitudes of PAGB A-F Stars in Galactic Globular Clusters

\begin{tabular}{|c|c|c|c|c|c|c|c|c|}
\hline Cluster & NGC & Star & $V$ & $B-V$ & Ref. $^{a}$ & $E(B-V)$ & $\begin{array}{c}\text { Distance } \\
(\mathrm{kpc})\end{array}$ & $M_{v}$ \\
\hline$\omega$ Cen & 5139 & ROA 24 & 10.80 & 0.36 & (1) & 0.11 & 5.2 & -3.1 \\
\hline$\ldots$ & $\begin{array}{c}5986 \\
\prime \prime\end{array}$ & $\begin{array}{l}\text { Bond } 1 \\
\text { Bond } 2\end{array}$ & $\begin{array}{l}12.48 \\
12.39\end{array}$ & $\begin{array}{l}0.72 \\
0.51\end{array}$ & $\begin{array}{l}(2) \\
\prime \prime\end{array}$ & $\begin{array}{c}0.25 \\
\prime \prime\end{array}$ & $\begin{array}{c}10.5 \\
\prime \prime\end{array}$ & $\begin{array}{l}-3.4 \\
-3.5\end{array}$ \\
\hline M19 & 6273 & ZNG 5 & 12.89 & 0.58 & (3) & 0.38 & $\begin{array}{l}10.6 \\
\text { Mea }\end{array}$ & $\begin{array}{r}-3.4 \\
-3.4 \\
0.2\end{array}$ \\
\hline
\end{tabular}

${ }^{a}$ Photometry references: (1) Cannon \& Stobie 1973; (2) Bond unpub.; (3) Harris et al. 1976. Reddenings and distances from Webbink 1985.

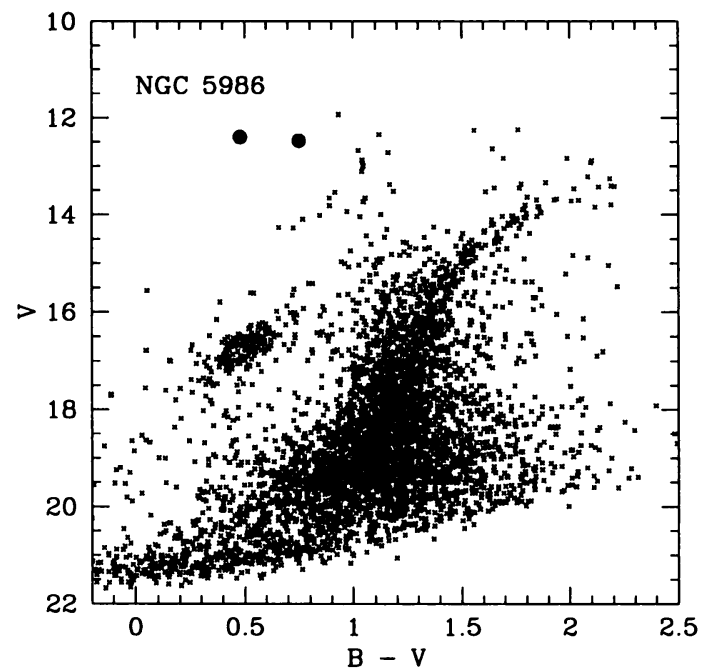

Figure 2. Preliminary CCD CMD for the Galactic globular cluster NGC 5986 (Bond, Fullton, Marois, \& O'Brien, in preparation). The large filled circles mark the two PAGB supergiants in this cluster. Their $V$ magnitudes agree within 0.09 mag.

diagram is shown in Fig. 2, with the two PAGB stars shown as large filled circles. They lie $4 \mathrm{mag}$ above the horizontal branch of the cluster, and are by far the brightest members of the cluster.

The route to the zero-point calibration for PAGB A and F stars is to begin with subdwarf parallaxes (which the Hipparcos data will soon be providing) in order to set the distance scale for Galactic globular clusters through main-sequence fitting. This will in turn set the absolute magnitudes 


\section{POST-AGB STARS AS STANDARD CANDLES}

for the PAGB stars. Four PAGB candidates are an insufficient number for a calibration, and it will be necessary to find more of them in globular clusters. We are now undertaking such a survey, using the technique described below. ¿From the fuel-consumption theorem, we expect to find PAGB stars in numbers roughly comparable to the number of Galactic Cepheids known in open clusters (Bond 1996).

\section{Discovery Technique}

Aside from an expected narrow LF, PAGB stars have another advantage: they are very easily recognized. Since they are of low mass but high luminosity, they have extremely low surface gravities. Around types A and F, the Balmer discontinuity is very sensitive to $\log g$, and thus the PAGB stars will have enormously large Balmer jumps.

The large jumps mean that stars of this type can be recognized easily through photometric techniques, if a filter whose bandpass is below the Balmer discontinuity is included. We are developing a hybrid photometric system, in which we combine the Gunn $u$ filter with the standard JohnsonCousins $B V I$ bandpasses.

\section{Potential Problems}

There are some important complications which may affect this proposed new candle, as discussed in more detail in Bond (1996). Probably the most important concern is the metallicity dependence of the absolute magnitudes. This is set by details of AGB mass loss that are still poorly understood. Nevertheless, the dependence may be small. Dopita et al. (1992) give interpolation formulae based on the Vassiliadis \& Wood (1994) theoretical PAGB tracks. This work predicts (with some extrapolation) that at a fixed age of $12 \mathrm{Gyr}$, going from $\log \left(Z / Z_{\odot}\right)=-1$ to -2 brightens $M_{\text {bol }}$ by 0.3 mag. Thus the effects of metallicity appear to be relatively small, and may even be calibratable.

\section{A Preliminary Test in the Halo of M31}

We have applied our $u B V I$ search technique in the halo of M31, using the Kitt Peak 4-m telescope and a $2048 \times 2048$ CCD which yields a $16^{\prime} \times 16^{\prime}$ field of view. We observed three fields at about $40^{\prime}-50^{\prime}$ from the nucleus along the minor axis, in which the surface density of M31 halo red giants is about 10,000 per CCD field. From the theoretical PAGB lifetimes we expect to find about 5 PAGB stars in each field.

We in fact identified 17 candidate objects in the three fields, having a large $(u-B)$ index with $0<B-V<0.5$. Their mean $V$ magnitude is 


\section{H. Bond}

21.1. If we adopt the Galactic calibration of $M_{V}=-3.4$, and a foreground reddening of $E(B-V)=0.08$, we find a distance modulus $(m-M)_{0}=24.2$. This is in superb agreement with the M31 distance of $24.3 \pm 0.1$ adopted in the review article by van den Bergh (1992), and gives us confidence that our method has promise.

\section{Future Plans}

Much work remains to be done in order to develop these potential excellent candles:

Primary calibration. We have begun a $u B V I$ survey of all of the Galactic globular clusters for PAGB stars. Once accurate subdwarf parallaxes become available from Hipparcos, we will have a firm calibration of the PAGB absolute magnitudes from the objects that we find in the clusters.

Local Group. Next we will search for PAGB stars in the Magellanic Clouds (for which extensive CTIO Curtis Schmidt and 1.5-m data are already in hand) and other members of the Local Group, to test and strengthen the $M_{V}$ calibration. The galaxies with Cepheids (the Clouds plus M31, M33, NGC 6822, etc.) will allow a direct confrontation with the Cepheid distance scale.

Intermediate distances. We will then be in a position to move out to intermediate-distance galaxies such as those of the Sculptor and M81 groups. At $V=23-25$, their PAGB stars should be reachable in about one night per field with a 4-m class telescope.

Virgo Cluster. Our ultimate aim will be to attain the distance of the Virgo Cluster, where the PAGB stars should have $V \approx 27.5$. This may be achievable with $H S T$ and its Advanced Camera, though the signal-to-noise will be low in a $u$-like filter.

\section{Summary}

1. Post-AGB A-F stars appear on theoretical and empirical grounds to be excellent candidates for standard candles.

2. They are the visually brightest members of Population II, and they are very easily recognized via $u B V I$ photometry, due to their extraordinarily large Balmer jumps.

3. Their absolute magnitudes can be calibrated in the Milky Way, using subdwarf parallaxes and globular clusters that contain PAGB stars.

4. We therefore argue that PAGB stars are the best available primary Population II candles. It should be possible to use them to reach the Virgo Cluster in just three steps: subdwarf parallaxes, Galactic globular clusters, and then directly to Virgo with HST. 


\section{POST-AGB STARS AS STANDARD CANDLES}

I gratefully acknowledge support by the NASA UV, Visible, and Gravitational Astrophysics Program, grant NAGW-4361. I thank Kitt Peak National and Cerro Tololo Interamerican Observatories for observing time, and my colleagues for wonderful discussions and hard work.

\section{References}

Aaronson, M., \& Mould, J. 1986, ApJ, 303, 1

Blöcker, T. 1995, A\&A, 299, 755

Blöcker, T., \& Schönberner, D. 1990, A\&A, 240, 11

Bond, H. E. 1977, BAAS, 9, 601

Bond, H. E. 1996, in The Extragalactic Distance Scale, ed. M. Livio \& M. Donahue, in press.

Cannon, R. D., \& Stobie, R. S. 1973, MNRAS, 162, 207

Dopita, M. A., Jacoby, G. H., \& Vassiliadis, E. 1992, ApJ, 389, 27

Harris, W. E., Racine, R., \& de Roux, J. 1976, ApJS, 31, 13

Jacoby, G. H. 1996, in The Extragalactic Distance Scale, ed. M. Livio \& M. Donahue, in press.

Paczynski, B. 1971, Acta Astr., 21, 417

Schönberner, D. 1983, ApJ, 272, 708

van den Bergh, S. 1992, PASP, 104, 861

Vassiliadis, E., \& Wood, P. R. 1994, ApJS, 92, 125

Webbink, R. F. 1985, in IAU Symposium 113, Dynamics of Star Clusters, ed. J. Goodman \& P. Hut, p. 541 